\title{
Multimodal treatment strategies for complex pediatric cerebral arteriovenous fistulas: contemporary case series at Barrow Neurological Institute
}

\author{
Hasan A. Zaidi, MD, M. Yashar S. Kalani, MD, PhD, Robert F. Spetzler, MD, \\ Cameron G. McDougall, MD, and Felipe C. Albuquerque, MD \\ Division of Neurological Surgery, Barrow Neurological Institute, St. Joseph's Hospital and Medical Center, Phoenix, Arizona
}

OBJECT Pediatric cerebral arteriovenous fistulas (AVFs) are rare but potentially lethal vascular lesions. Management strategies for these lesions have undergone considerable evolution in the last decade with the advent of new endovascular, surgical, and radiosurgical technologies. This study sought to review current treatment strategies and long-term clinical outcomes at a high-volume cerebrovascular institute.

METHODS A retrospective chart review was performed on patients with a diagnosis of cerebral AVF from 1999 to 2012. Patients with carotid-cavernous fistulas, vein of Galen malformations, and age $>18$ years were excluded from final analysis. Medical history, surgical and nonsurgical treatment, and clinical outcomes were documented. Pre- and postoperative angiograms were analyzed to assess for obliteration of the fistula.

RESULTS Seventeen patients with pial AVFs (29.4\%), dural AVFs (64.7\%), or mixed pial/dural AVFs (5.9\%) were identified. The majority of lesions were paramedian $(70.6 \%)$ and supratentorial $(76.5 \%)$. The study population had a mean age of 6.4 years, with a slight male predominance $(52.9 \%)$, and the most common presenting symptoms were seizures $(23.5 \%)$, headaches $(17.6 \%)$, congestive heart failure $(11.7 \%)$, and enlarging head circumference (11.7\%). Among patients who underwent intervention $(n=16), 56.3 \%$ were treated with endovascular therapy alone, $6.3 \%$ were treated with open surgery alone, and $37.5 \%$ required a multimodal approach. Overall, $93.8 \%$ of the treated patients received endovascular treatment, $43.8 \%$ received open surgery, and $12.5 \%$ received radiosurgery. Endovascular embolysates included Onyx (n $=5), N$-butyl cyanoacrylate (NBCA; $n=4)$, or coil embolization $(n=7)$ with or without balloon assistance $(n=2)$. Complete angiographic obliteration was achieved in $87.5 \%$ at the last follow-up evaluation (mean follow-up 3.1 years). One infant with incomplete AVF obliteration died of congestive heart failure, and 1 patient with complete obliteration died of acute sinus thrombosis, with an overall complication rate of $18.8 \%$.

CONCLUSIONS Pediatric cerebral AVFs are challenging neurosurgical lesions. Although advancements in endovascular therapy in the last decade have greatly changed the natural course of this disease, a multidisciplinary approach remains necessary for a large subset of patients. Surgeon experience with a thorough analysis of preoperative imaging is paramount to achieving acceptable clinical outcomes.

http://thejns.org/doi/abs/10.3171/2014.11.PEDS14468

KEY WORDS arteriovenous fistula; dural; endovascular; multimodal; pial; stereotactic radiosurgery; Onyx; NBCA; vascular disorders

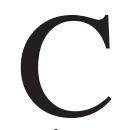
EREBRAL arteriovenous fistulas (AVFs) are potentially lethal vascular lesions characterized by an abnormal connection between an artery and vein without an intervening capillary channel or nidus. ${ }^{14}$ Feeding arteries derived from pial or cortical vessels are termed pial AVFs and those derived from dural vessels are termed dural AVFs. Although rare in the pediatric population, $, 18,19$ cerebral AVFs can result in death from highoutput cardiac failure, developmental delay, or cognitive decline from obstructive hydrocephalus. ${ }^{18}$ Direct arterial pulsations into compliant venous channels can result in the formation of a giant venous varix, frequently causing

ABBREVIATIONS ACA = anterior cerebral artery; $\mathrm{AP}=$ anteroposterior; $\mathrm{AVF}=$ arteriovenous fistula; $\mathrm{AVM}=$ arteriovenous malformation; $\mathrm{DSA}=$ digital subtraction angiography; ECA = external carotid artery; GKS = Gamma Knife surgery; GOS = Glasgow Outcome Scale; ICA = internal carotid artery; MMA = middle meningeal artery; NBCA = $\mathrm{N}$-butyl cyanoacrylate; $\mathrm{OA}=$ occipital artery; $\mathrm{PCA}=$ posterior cerebral artery; $\mathrm{STA}=$ superficial temporal artery; $\mathrm{VA}=$ vertebral artery.

SUBMITTED September 4, 2014. ACCEPTED November 24, 2014.

INCLUDE WHEN CITING Published online March 27, 2015; DOI: 10.3171/2014.11.PEDS14468.

DISCLOSURE Dr. McDougall is a consultant to Covidien (maker of Onyx), Codman (maker of NBCA), and Microvention. 
focal neurological deficits or seizures from mass effect or venous infarcts. Unlike cerebral arteriovenous malformations (AVMs), pial or dural AVFs do not require resection. ${ }^{9}$ Rather, disconnection at the fistulous point via endovascular, surgical, or radiosurgical means can result in long-term cure with eventual regression of the associated venous varices. ${ }^{9}$ Treatment paradigms have evolved tremendously over the last few decades, and they parallel the technological innovations introduced in neurosurgery. Early reports of cure in the adult literature were heavily dominated by surgical means, including resection of a varix by Antunes et al. in $1977^{1}$ and surgical clipping by Drake in $1979 .{ }^{4}$ As surgeons accumulate experience with novel endovascular embolysates, they are increasingly using balloon occlusion, ${ }^{17}$ platinum coils, ${ }^{7}$ or $N$-butyl cyanoacrylate (NBCA; Codman) ${ }^{2}$ to obliterate fistulas. Furthermore, radiosurgery has shown promising results in a small subset of patients ${ }^{6,10}$ A review of modern treatment options and clinical outcomes for pediatric cerebral AVFs is lacking, with recent articles often consolidating a multidecade experience ${ }^{8,20}$ or showing heavy bias toward endovascular therapy alone. ${ }^{12,18,19}$ We sought to review the treatment methodology and clinical outcomes of our institution's multidisciplinary approach to treating these complex cerebrovascular lesions in the modern era.

\section{Methods}

A retrospective review of our prospectively maintained database was performed on patients diagnosed with a cerebral AVF from 1999 to 2012. Only patients with preinterventional angiograms documenting a pial or dural AVF associated with a large venous varix were included because these are known to be poor prognostic indicators. ${ }^{5}$ Dedicated angiograms of the internal carotid, external carotid, and bilateral vertebral arteries (VAs) were analyzed. Patients with carotid-cavernous fistulas, vein of Galen malformations, and ages $>18$ years were excluded from final analysis. Medical history, surgical and nonsurgical treatment, and clinical outcomes were documented. All available postoperative angiograms were analyzed to assess for obliteration of the fistula. In-hospital charts, clinic notes, and imaging studies were carefully reviewed to determine all additional surgical and nonsurgical interventions. Pre- and postintervention Glasgow Outcome Scale (GOS) scores and GOS scores at last follow-up evaluation were obtained.

\section{Results}

Between 1999 and 2012, a total of 21 patients were referred to our institution with documented evidence of a cerebral dural AVF on formal angiographic studies. Of these 21 patients, 4 did not demonstrate an associated large venous varix and were excluded from final analysis. A total of 17 patients were part of the final analysis; this study population had an average age of 6.4 years, and the majority (52.9\%) were male (Table 1). Three patients had a known genetic syndrome, including trisomy 21, Klippel-Trenaunay-Weber syndrome, and BannayanRiley-Ruvalcaba syndrome. All preinterventional 4-vessel angiograms were reviewed and patients were confirmed to have either a pial AVF $(n=5)$, a dural $\operatorname{AVF}(n=11)$, or mixed pial/dural AVF $(\mathrm{n}=1)$. The majority of lesions were paramedian $(70.6 \%)$ and supratentorial $(76.5 \%)$. The primary feeding vessel was most commonly derived from the anterior circulation (52.9\%), followed by the posterior circulation (41.2\%) and the external carotid artery (ECA; $35.3 \%$ ). The most common presenting symptoms were seizures $(23.5 \%)$, headaches (17.6\%), congestive heart failure (11.7\%), and enlarging head circumference (11.7\%). None of the patients in our series experienced a hemorrhage during the interval from diagnosis to treatment.

Of the 17 patients in our series, 16 (94.1\%) ultimately received treatment and 1 patient (5.9\%) elected to undergo close observation. Overall, complete angiographic obliteration at last follow-up was achieved in 87.5\% (14/16; average 3.1 years). One patient (6.3\%) was treated with open surgery alone, but more than one-third of the patients (n $=6,37.5 \%$ ) required a multimodal approach in treating these lesions. Among these 6 patients, treatment modalities included open surgical clipping (100\%), endovascular embolization $(100 \%)$, or radiosurgery (33.3\%). Overall, the majority of patients were treated with endovascular therapy alone (56.3\%), and endovascular embolysates including Onyx (Covidien; $n=5)$, NBCA $(n=4)$, or coil embolization $(\mathrm{n}=7)$ with or without balloon assistance $(\mathrm{n}=2)$. We found that patients treated with endovascular surgery during the early part of our series (1999-2008) primarily received coil embolization, whereas those in the more recent set (2010-2012) were treated primarily with either NBCA or Onyx embolization. As our experience in handling and predicting the course of liquid embolysates increased, we found that it allowed for a more targeted placement of the embolic agent into the proximal portion of the draining vein without compromising surrounding normal vasculature.

The overall complication rate for treated patients in our series was $18.8 \%$, including 1 cardiovascular-related death $(6.3 \%), 1$ procedure-related death $(6.3 \%)$, and 1 patient who required reoperation for a pseudomeningocele repair after a craniotomy (6.3\%; Table 1). The first of these patients (Case 6, Table 1) was a neonate who presented with congestive heart failure and evidence of bilateral high-flow dural AVFs with multiple feeders from the posterior circulation and external carotid circulation. She was treated with 5-stage Onyx embolization, resulting in significant reduction of flow through the fistula but with incomplete obliteration. She ultimately died of congestive heart failure at 1 month of age despite maximal medical treatment. The second patient (Case 13, Table 1) presented with seizures and was noted on diagnostic angiography to have a pial AVF fed primarily from $A_{1}$ to the basal vein of Rosenthal. Temporary balloon occlusion clearly identified the fistulous point, but due to the high-flow nature of this fistula, it was believed to be too dangerous to treat using endovascular embolysates. The patient subsequently underwent an uncomplicated bifrontal craniotomy for microsurgical clipping with an intraoperative angiogram demonstrating complete obliteration of the fistulous connection. The patient initially did well, but was noted on postoperative Day 1 to have visual decline. An immediate angiogram demonstrated acute transverse sinus thrombo- 


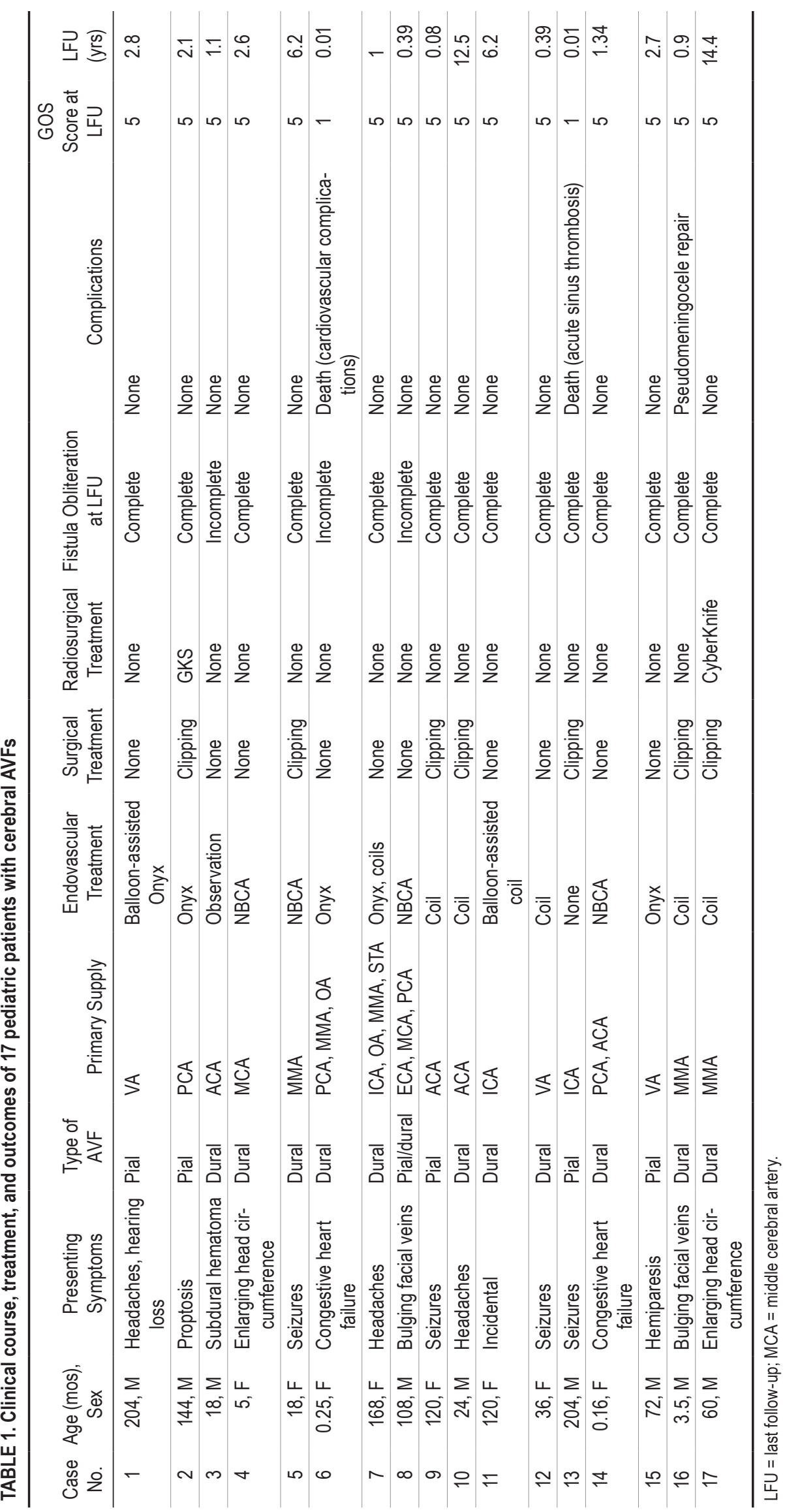


sis with expansion of the venous varix, resulting in optic nerve compression. Despite endovascular thrombectomy, reopening of the craniotomy for optic nerve decompression, and external ventricular drain placement, the patient developed intractable elevated intracranial pressures and suffered global cerebral hypoxia. He was declared brain dead on postoperative Day 4.

Two lessons were learned from this case. First, caution must be exercised in the setting of high-flow fistulas on preinterventional angiograms. Abrupt obliteration of high-flow cerebral AVFs instantaneously alters cerebral hemodynamics, which can result in rapid decompensation from a sinus thrombosis. In this situation, a detailed conversation must take place with the patient and the family concerning the risk of observation versus the high risk of perioperative complications. Second, any clinical suspicion of venous/sinus thrombosis should be treated early and aggressively with intravenous anticoagulation unless otherwise contraindicated. This suspicion includes subtle neurological deficits, as our patient in this case experienced (visual decline).

\section{Illustrative Cases Case 1}

A 17-year-old boy presented with altered mental status to an outside hospital and was found to have an intraparenchymal hemorrhage. A formal angiogram demonstrated a high-flow pial AVF fed primarily from the right VA (Fig. $1 \mathrm{~A}-\mathrm{C})$. The patient underwent an unsuccessful subtotal embolization at an outside facility and transferred to our institution for definitive management. On admission, he had some mild right-sided hearing loss but was otherwise neurologically intact. Upon analysis of the formal angiogram, a feeding arterial pedicle from the $V_{4}$ segment of the VA was identified. A HyperForm balloon (Covidien) was used to safely infuse Onyx-34 at the fistulous point while reducing the risk of unintended embolization of normal posterior fossa vasculature. The patient developed no new clinical deficits after embolization (Fig. 1D-F), and postprocedural digital subtraction angiography (DSA) as well as the last follow-up DS angiogram at 2.8 years demonstrated no recurrent filling of the fistula (Fig. $1 \mathrm{G}$ and $\mathrm{H}$ ).

\section{Case 2}

A 12-year-old boy was referred to our neurovascular service after workup for sudden, 2-day history of persistent headaches and left-eye proptosis, which revealed a large left occipital vascular lesion. As previously described by our group,${ }^{15}$ vascular imaging of the lesion revealed a $4 \times 3.7-\mathrm{cm}$ non-Galenic AVF supplied by the left posterior cerebral artery (PCA; Fig. 2A and B). Venous drainage distal to the aneurysmal sac occurred through a large dilated vein of Galen and straight sinus with subsequent congestion of the vein of Labbé. The treatment plan consisted of angiography with possible embolization followed by microsurgical treatment if necessary.

The patient underwent cerebral angiography under general anesthesia for delineation of the angioarchitecture and treatment of his lesion. Angiography revealed a highflow AVF fed by the left PCA and drained by the vein of
Galen (Fig. 2C and D). The fistulous point was approached via a transarterial route and embolized with a combination of Onyx-18 and Onyx-34 using balloon assistance (Fig. $2 \mathrm{E}-\mathrm{G})$. During the procedure we attempted unsuccessfully to use adenosine to induce temporary cardiac arrest during the injection of the embolysate. The posttreatment angiograms revealed a near-complete embolization of the fistula with trace flow through the fistulous point (Fig. $2 \mathrm{G})$. At this point it was believed that the residual fistula would likely thrombose spontaneously. The patient was kept intubated and returned to the intensive care unit with strict blood pressure control. Postprocedurally, the patient remained neurologically grossly intact with minimal visual complaints. Magnetic resonance imaging confirmed the embolization of the fistula with minimal adjacent restricted diffusion in the left occipital lobe. On postoperative Day 2 the patient was returned to the angiography suite to confirm complete obliteration of the fistula.

Repeat cerebral angiography 2 days after embolization revealed a primarily thrombosed venous pouch, but continued supply to the AVF via distal PCA branches was evident (Fig. 3A and B). Attempts to embolize the residual feeders via both transarterial and transvenous routes were unsuccessful. Given the continued flow into the fistula we elected to pursue microsurgical obliteration of the residual fistula.

The following day the patient was taken to the operative theater for microsurgical disconnection of the residual fistula. Intraoperatively, the large varix was dissected, identifying the large PCA feeder that was embolized with Onyx. The fistulous point was carefully exposed and a permanent aneurysm clip was placed across the PCA feeder distal to the embolysate. Several small branches were coagulated and cut. Ensuring that the fistulous connection was completely obliterated required the distal venous outflow to be temporarily occluded and the varix to be opened. We removed Onyx and the thrombus from within the varix and did not observe flow into the varix, thereby confirming complete obliteration of the fistula.

The patient was taken to the angiography suite postoperatively to document complete occlusion of the fistula. Postoperative angiography revealed complete disconnection of the fistula without any residual inflow or early draining vein (Fig. 3C and D). The patient was neurologically intact, with continued mild visual complaints. He was discharged to a neurological rehabilitation facility on postoperative Day 3 after microsurgical treatment of the fistula.

Despite significant improvement in symptoms, the patient returned 3 months after treatment with new-onset headaches. Magnetic resonance imaging evaluation revealed thrombosis of the lesion (Fig. 4A and B) with improvement of T2 signal abnormality within the occipital lobe. Given the patient's symptoms and the sudden onset nature of his complaint, we elected to perform a diagnostic angiogram to further evaluate the treated fistula. Formal angiography revealed a small early draining nidus fed by a small distal branch of the left PCA that was believed to be inappropriate for endovascular treatment (Fig. 4CF). This lesion appeared very different from the original pathology noted on the angiogram. Two possibilities may 

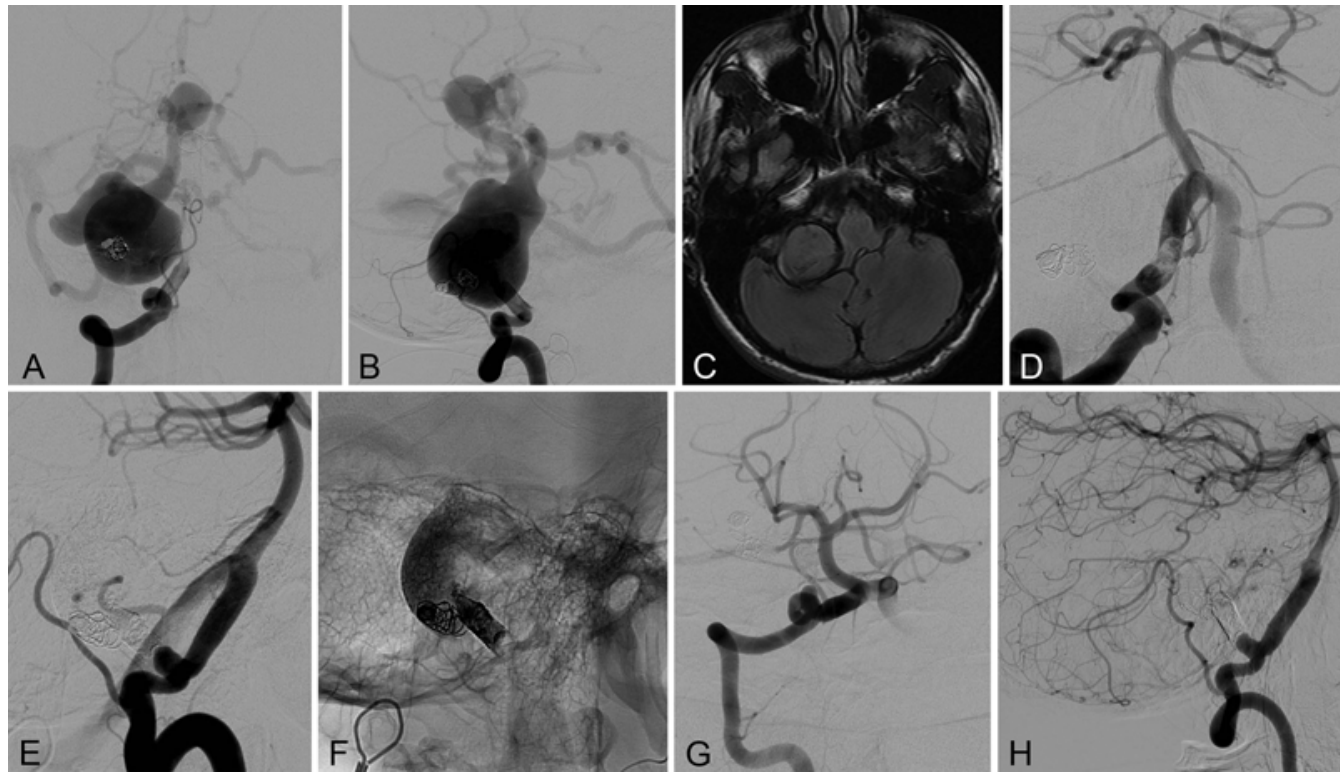

FIG. 1. Case 1. Images obtained in a 17-year-old boy who presented to an outside hospital with a new intraparenchymal hemorrhage and was found to have a pial AVF fed primarily from the right $V_{4}$ segment as demonstrated on right VA injection on anteroposterior (AP; A) and lateral (B) DSA. There was evidence of significant mass effect from ectatic vessels on axial T2-weighted FLAIR MRI (C). This mass effect was unsuccessfully treated with coil embolization at an outside facility and the patient was then transferred to our institution. He underwent a single-stage, balloon-assisted Onyx-34 embolization of this fistula with complete obliteration of the fistula on postoperative right VA injection on AP (D) and lateral (E) DSA. Lateral plain radiographs (F) demonstrated the Onyx cast within the feeding pedicle as well as the coil within the fistulous bulb. The last follow-up evaluation at 2.8 years demonstrated no residual fistula on right VA injection on AP (G) and lateral (H) DSA.

explain this finding: 1) the original embolization procedure may have unmasked a concomitantly present nidal AVM, or 2) altered cerebral hemodynamics with incomplete obliteration of a giant AVF may have resulted in the congestion of cortical vessels over the course of 3 months.
Given the size and location of the nidus, we believed that this lesion was also a high risk for microsurgical treatment with a high risk of neurological compromise from the approach. After a multidisciplinary discussion, we elected to treat the residual lesion with stereotactic radiosurgery.
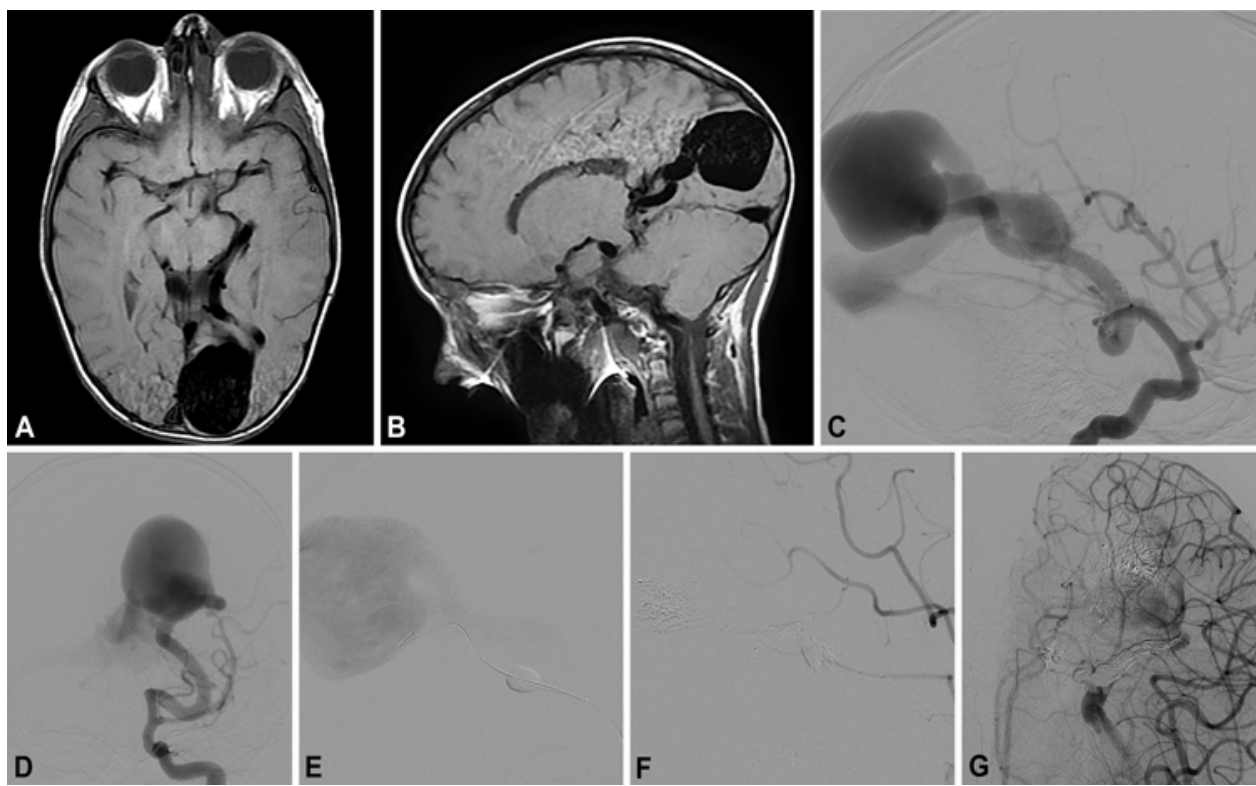

FIG. 2. Case 2. Axial (A) and sagittal (B) T1-weighted MRI reveals a large area of flow void within the left occipital lobe concerning for a vascular malformation. Lateral (C) and AP (D) angiography of the left ICA reveals a non-Galenic AVF fed by the left PCA and a large varix drained by the vein of Galen. Using a balloon-assisted technique (E), we performed a transarterial embolization of the fistulous point using a combination of Onyx-18 and Onyx-34 (F). A postembolization AP view of the left ICA injection (G) reveals minimal residual filling of the fistula. 

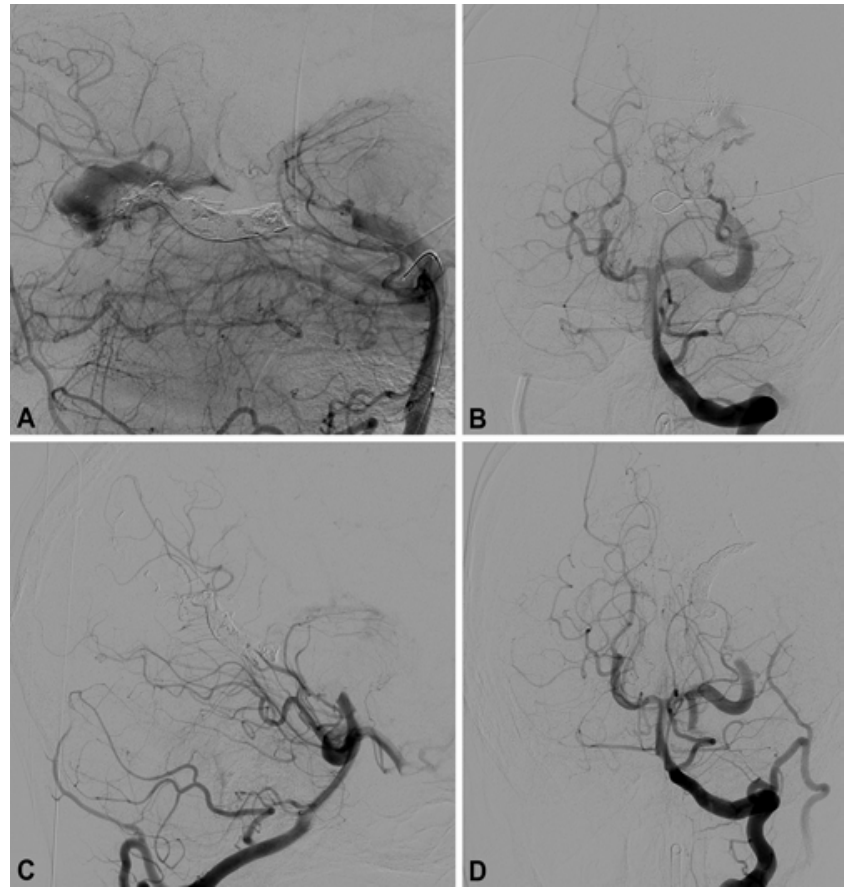

FIG. 3. Case 2. Lateral (A) and AP (B) VA injections demonstrate residual filling of the AVF by distal small PCA branches. It was believed that the residual AVF could not be safely treated using endovascular techniques and the patient was taken to the operative theater for microsurgical obliteration of the fistula. Postoperative lateral (C) and AP (D) VA injections demonstrate complete obliteration of the fistula.
Given the small size of this lesion and the young age of the patient, we decided that erring on the lower end of an effective dose range of 15-26 Gy would be efficacious without exposing a child to unnecessary radiation. ${ }^{16,21}$ The patient was treated with Gamma Knife surgery (GKS; Elekta $\mathrm{AB}$ ) and received $17 \mathrm{~Gy}$ at the $50 \%$ isodose line (Fig. 5).

Eighteen months after radiosurgery the patient is at his baseline neurological level without symptoms concerning for fistula recurrence. He awaits his postradiosurgical treatment follow-up evaluation that is scheduled for 3 years from the time of stereotactic radiosurgery.

\section{Case 7}

A 14-year-old girl with a history of Bannayan-RileyRuvalcaba syndrome and a history of chronic debilitating headaches was treated at an outside facility with Chiari decompression and lumboperitoneal shunt placement. She presented to our facility for a venogram and arteriogram for suspected pseudotumor cerebri and was found to have a complex superior sagittal sinus dural AVF with multiple parasitized pial branches of the anterior cerebral artery (ACA) and cavernous internal carotid artery (ICA), as well as the middle meningeal artery (MMA), superficial temporal artery (STA), and occipital artery (OA) all contributing to the fistula (Fig. 6A and B). As previously described by our group,${ }^{13}$ the patient underwent a 2 -stage procedure, first with transvenous balloon-assisted Onyx embolization via 2 distinct MMA branches accessed via the venous pouch. Second, a transarterial route via the MMA branches with Onyx embolization bolstered by coil embolization further reduced flow via the fistula (Fig. 6C and D). Immediate postoperative DSA demonstrated a residual fistula fed primarily from the MMA and STA (Fig. 6E). Given
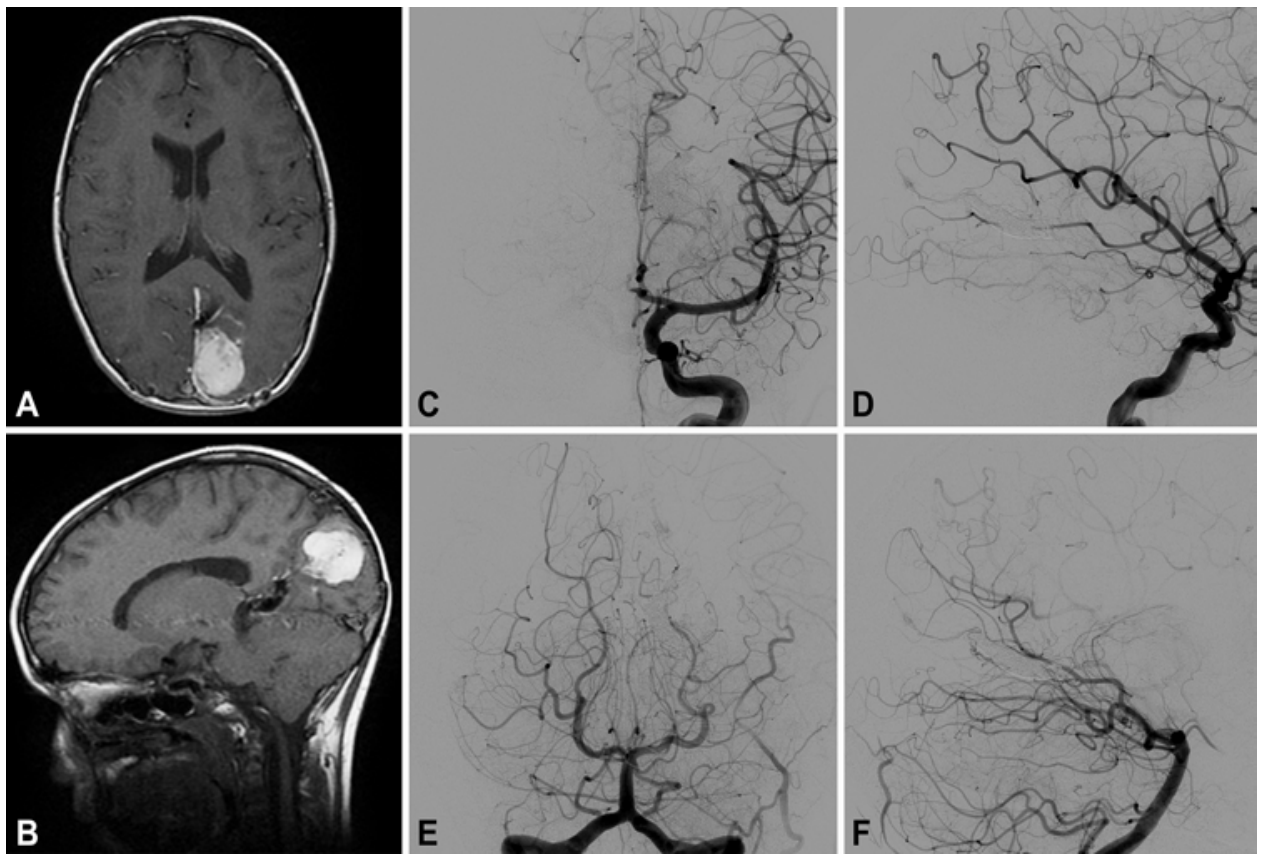

FIG. 4. Case 2. Follow-up axial (A) and sagittal (B) T1-weighted MRI obtained 3 months postoperatively demonstrates a thrombosed varix without obvious residual fistula. Anteroposterior (C) and lateral (D) left ICA injections and AP (E) and lateral (F) VA injections demonstrate a small early draining nidus fed by a small distal branch of the left PCA that was believed to be inappropriate for endovascular treatment. 


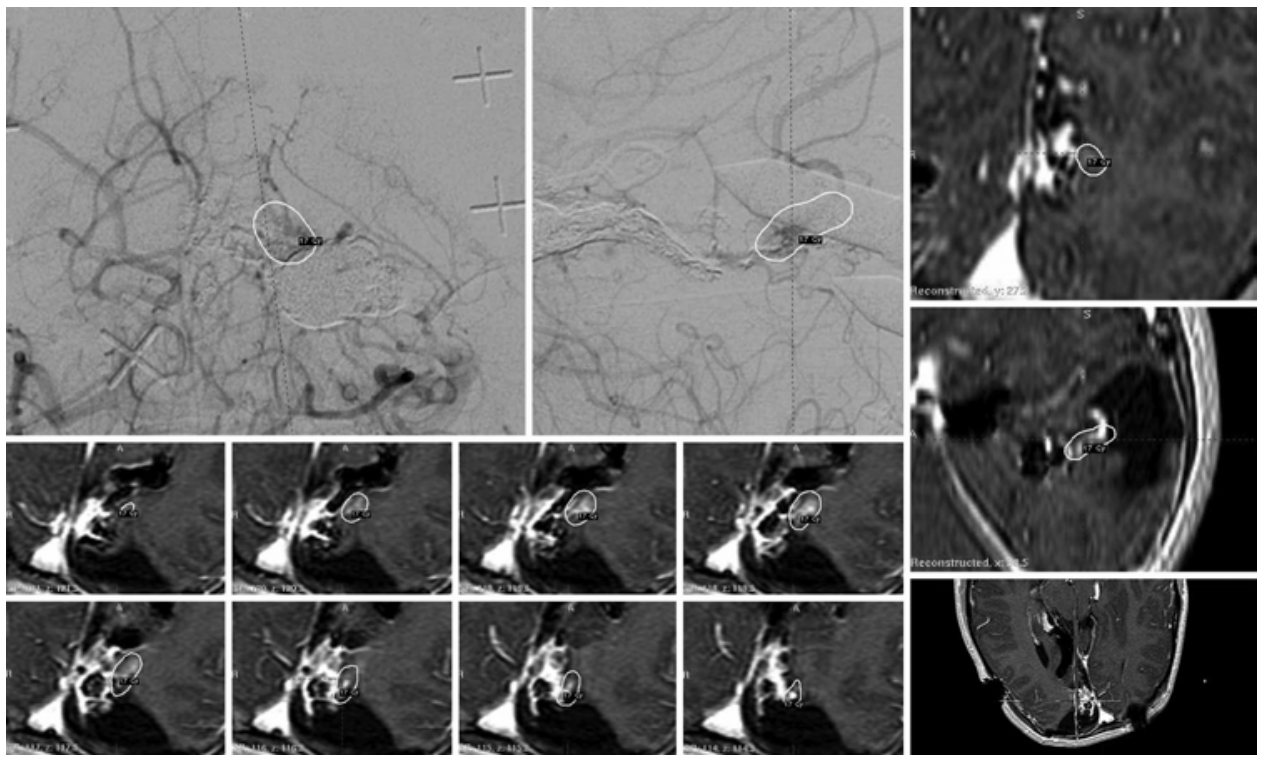

FIG. 5. Case 2. The GKS treatment plan for the residual non-Galenic fistula. The patient received 17 Gy to the $50 \%$ isodose line.

that the patient was doing well clinically, we decided to closely monitor this residual fistula with serial imaging. Three-month follow-up and the last follow-up DS angiogram demonstrated complete angiographic obliteration of the fistula (Fig. 6F).

\section{Discussion}

Pediatric cerebral AVFs are complex lesions that necessitate a multimodal approach to therapy. In the era of modern endovascular surgery (1999-2012), 56.3\% of patients in this case series were treated with endovascular therapy alone but $37.5 \%$ required a multimodal approach at our high-volume neurovascular institute. Weighing the risks and advantages of a particular treatment approach in light of the anatomical configuration of the fistulas is paramount, and neurosurgeons must be proficient in recognizing the limitations of each modality to maximize clinical results. Evaluation by a multidisciplinary team proficient in open, endovascular, and radiosurgical techniques is necessary to effectively treat these complex lesions in the pediatric population and achieve acceptable outcomes (Fig. 7).

\section{Open Versus Endovascular Treatment}

The preferred method of treatment for cerebral AVFs has closely paralleled the technological advances introduced into neurovascular surgery. Prior to the refinement of endovascular techniques and development of modern embolysates, treatment of cerebral AVFs was primarily dominated by open surgical disconnection. In a literature review by Hoh et al. ${ }^{9}$ of 79 adult and pediatric patients with pial single-channel AVFs between 1970 and 2000, endovascular treatment was attempted in $63.3 \%$. However, nearly $40 \%$ of these patients had failed treatment or required additional treatment. ${ }^{11}$ Conversely, of the $64.6 \%$ of patients who received open treatment, $92.2 \%$ achieved angiographic obliteration. As the cumulative experience among neuroendovascular surgeons in treating complex fistulas has increased, the pendulum has recently swung in favor of endovascular surgery. In a literature review of 147 pediatric pial AVFs treated between 1977 and 2012, Madsen et al. found that 19 of 23 reports of surgical interventions were published prior to $2002 ; ;^{12}$ of their 6 reported cases, all were treated with endovascular therapies alone. ${ }^{8}$ Similarly, Lv et al. noted in a series of 10 pediatric pial AVFs treated between 1998 and 2008 that endovascular therapies were considered the primary mode of treatment in all cases and open surgical treatment was discouraged. ${ }^{11}$ This paradigm shift highlights the strides endovascular technologies have made in the last decade, but should not undermine the utility of open surgical options for a significant subset of patients.

Unlike adult fistulas, pediatric cerebral AVFs are often high-flow lesions with aberrant anatomy, increasing the risk of iatrogenic injury during microcatheter access to the fistula and unintended deployment of embolysate into normal vessels. Small patient size can complicate femoral access, and limits the amount of injectable contrast. Furthermore, ionizing radiation can have deleterious longterm effects in the neonate. Complex lesions often require multistaged therapies with a prolonged period of general anesthesia, furthering the risk of medical complications in a fragile neonate with little reserve. Hetts et al. reported a complication rate of $60 \%$ among 25 pial AVFs in pediatric patients treated between 1986 and 2011, including both major complications (such as femoral artery transection and basilar artery perforation) and minor complications (such as lower-extremity embolus and microcatheteror microwire-induced vascular perforations). ${ }^{8}$ Similarly, Madsen et al. reported a complication rate of $60 \%$ in a series of 5 pial AVFs in pediatric patients treated with endovascular embolization between 2000 and 2012. ${ }^{12}$ Although this indicates that endovascular obliteration methods can potentially carry a significant amount of risk, open surgical options have their own set of disadvantages. A large number of lesions are located in deep, eloquent territory, and surgical access and visualization can be limited by normal brain tissue, as well as the large varix often associ- 

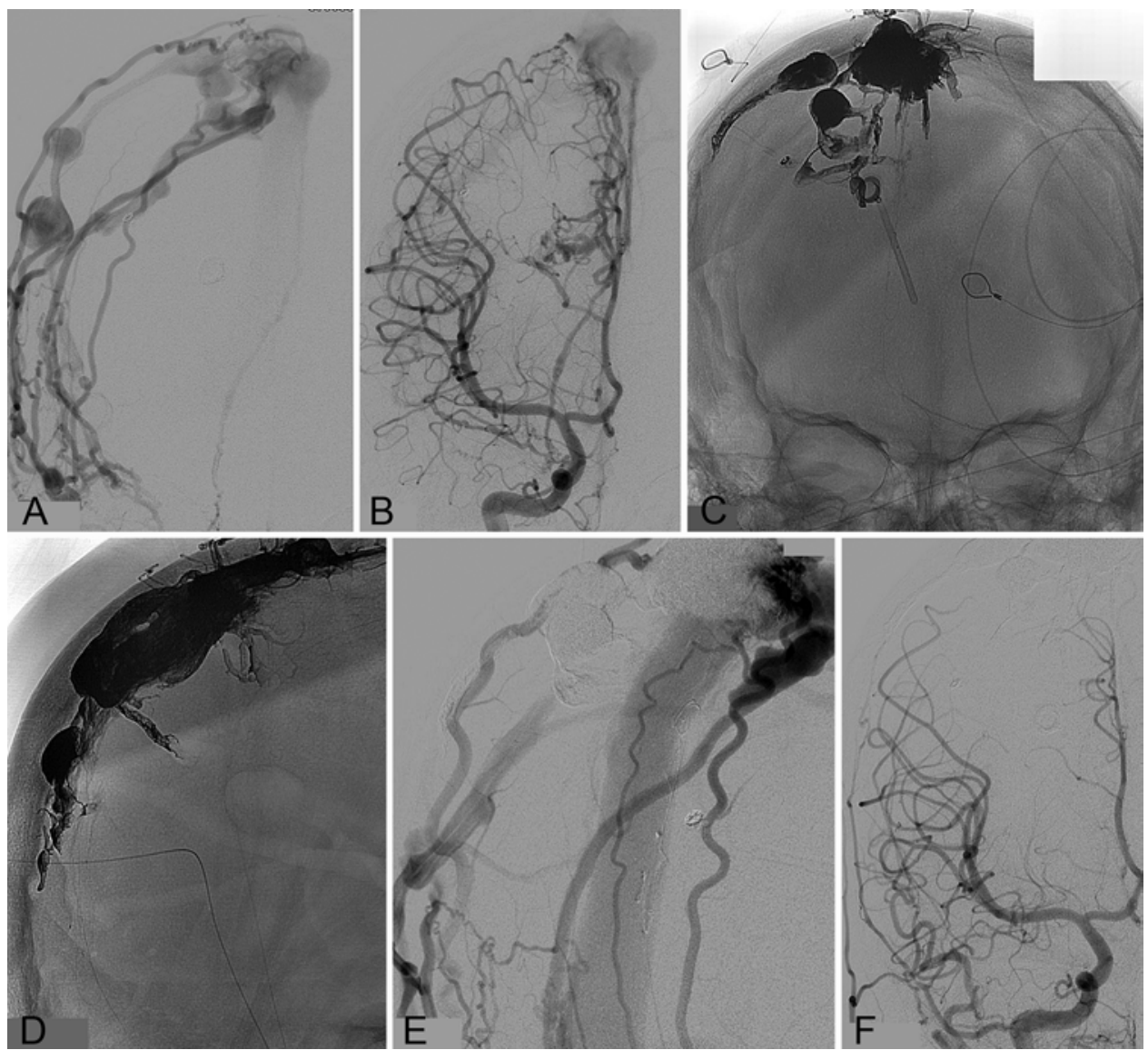

FIG. 6. Case 7. Images obtained in a 14-year-old girl who presented with chronic debilitating headaches. On right ECA lateral injection (A) and right ICA AP injections (B), the patient was found to have a complex superior sagittal sinus dural AVF with multiple parasitized pial branches of the ACA and cavernous ICA, as well as the MMA, STA, and OA all contributing to the fistula. A 2-stage transarterial/transvenous Onyx embolization procedure was performed with AP (C) and lateral (D) skull radiographs demonstrating a large Onyx cast with reduced flow via the fistula. The residual fistula fed primarily from the MMA and STA, as noted on the right ECA AP DSA (E). Given that the patient was doing well clinically, we decided to closely monitor this residual fistula with serial imaging. Three-month follow-up and last follow-up DSA demonstrated complete angiographic obliteration of the fistula on right common carotid artery AP radiographs (F), with angiographic confirmation of fistula obliteration at last follow-up (1 year).

ated with these lesions. ${ }^{11}$ Unlike AVMs in which a clear nidus is often easily identifiable, the fistulous points in cerebral AVFs are more difficult to identify and the preoperative angiography is often hard to translate to direct intraoperative anatomy. As previously discussed, we experienced 1 catastrophic periprocedural complication with open surgical clip placement due to acute thrombosis of the transverse sinus. This is a potential complication any time a high-flow fistula is ligated and not necessarily related to the mode of treatment itself.

\section{Stereotactic Radiosurgery}

Radiosurgery has shown some efficacy in the adult population with cerebral AVFs when used as adjunctive treatment. In the largest series by Cifarelli et al., ${ }^{3} 55$ adults with dural AVFs were treated with GKS from 1989 to 2005 and angiographic obliteration was achieved in 54\%-65\% at 3 years. However, $5 \%$ of patients experienced a hemorrhage during the latency period from treatment to obliteration. Although these results show radiosurgery to be promising as an adjunctive treatment for refractory cases in the adult literature, the pediatric neurosurgical literature in support of radiosurgery is scarce. Prior to our report, Weon et al. were the only authors to report successful treatment of a pediatric dural AVF with radiosurgery and embolization. ${ }^{20}$ Hetts et al. ${ }^{8}$ recommended that radiosurgery be reserved only for pediatric patients with AVFs and a concomitant AVM nidus, and suggested that radiosurgery was unlikely to induce regression of larger caliber fistulas. In our series, we treated 2 patients with endovascular embolization, surgical clip placement, and radiosurgery, with evidence of complete obliteration at last follow-up evaluation. Of these 2 patients, an older patient (Case 2, Table 1) was treated with GKS and a younger patient (Case 17, Table 1) was treated with the CyberKnife (Accuray) because his calvaria was too small to fit a Leksell frame. Further studies are necessary to understand the risks and benefits of radiosurgery in this subset of patients.

\section{Conclusions}

Pediatric cerebral AVFs are some of the most challenging lesions faced by neurovascular surgeons. Although 


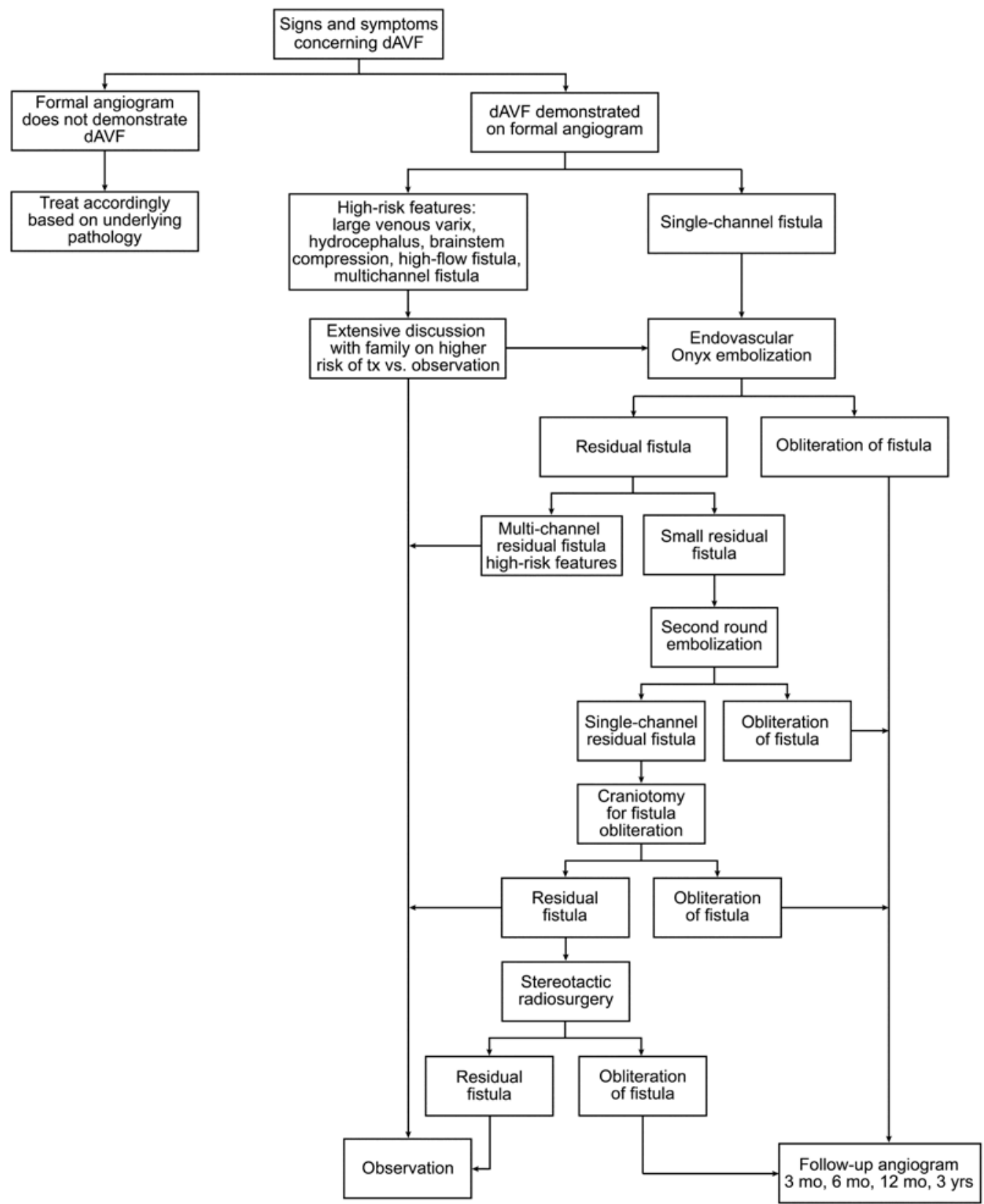

FIG. 7. Flow diagram demonstrating the treatment (tx) algorithm for pediatric dural AVFs (dAVFs).

advancements in endovascular therapy in the last decade have greatly changed the natural course of this disease, a multidisciplinary approach is still necessary for a large subset of patients. A clear understanding of the limits of each treatment modality, as well as thorough analysis of preoperative imaging, is paramount to achieving acceptable clinical outcomes.

\section{References}

1. Antunes JL, DiGiacinto GV, Michelsen WJ: Giant hemispheric arteriovenous fistula in an infant. Surg Neurol 7:45-48, 1977

2. Barnwell SL, Ciricillo SF, Halbach VV, Edwards MS, Cogen PH: Intracerebral arteriovenous fistulas associated with intra- parenchymal varix in childhood: case reports. Neurosurgery 26:122-125, 1990

3. Cifarelli CP, Kaptain G, Yen CP, Schlesinger D, Sheehan JP: Gamma knife radiosurgery for dural arteriovenous fistulas. Neurosurgery 67:1230-1235, 2010

4. Drake CG: Cerebral arteriovenous malformations: considerations for and experience with surgical treatment in 166 cases. Clin Neurosurg 26:145-208, 1979

5. Duffau H, Lopes M, Janosevic V, Sichez JP, Faillot T, Capelle L, et al: Early rebleeding from intracranial dural arteriovenous fistulas: report of 20 cases and review of the literature. $\mathbf{J}$ Neurosurg 90:78-84, 1999

6. Fabrikant JI, Lyman JT, Hosobuchi Y: Stereotactic heavy-ion Bragg peak radiosurgery for intra-cranial vascular disorders: method for treatment of deep arteriovenous malformations. Br J Radiol 57:479-490, 1984 
7. Halbach VV, Higashida RT, Hieshima GB, Hardin CW, Dowd CF, Barnwell SL: Transarterial occlusion of solitary intracerebral arteriovenous fistulas. AJNR Am J Neuroradiol 10:747-752, 1989

8. Hetts SW, Keenan K, Fullerton HJ, Young WL, English JD, Gupta N, et al: Pediatric intracranial nongalenic pial arteriovenous fistulas: clinical features, angioarchitecture, and outcomes. AJNR Am J Neuroradiol 33:1710-1719, 2012

9. Hoh BL, Putman CM, Budzik RF, Ogilvy CS: Surgical and endovascular flow disconnection of intracranial pial singlechannel arteriovenous fistulae. Neurosurgery 49:1351-1364, 2001

10. Hosobuchi Y, Fabricant J, Lyman J: Stereotactic heavy-particle irradiation of intracranial arteriovenous malformations. Appl Neurophysiol 50:248-252, 1987

11. Lv X, Li Y, Jiang C, Wu Z: Endovascular treatment of brain arteriovenous fistulas. AJNR Am J Neuroradiol 30:851856,2009

12. Madsen PJ, Lang SS, Pisapia JM, Storm PB, Hurst RW, Heuer GG: An institutional series and literature review of pial arteriovenous fistulas in the pediatric population. Clinical article. J Neurosurg Pediatr 12:344-350, 2013

13. Moon K, Ducruet AF, Crowley RW, Klas K, Bristol R, Albuquerque FC: Complex dural arteriovenous fistula in Bannayan-Riley-Ruvalcaba syndrome. J Neurosurg Pediatr 12:87-92, 2013

14. Nelson K, Nimi Y, Lasjaunias P, Berenstein A: Endovascular embolization of congenital intracranial pial arteriovenous fistulas. Neuroimaging Clin N Am 2:309-317, 1992

15. Newman CB, Hu YC, McDougall CG, Albuquerque FC: Balloon-assisted Onyx embolization of cerebral single-channel pial arteriovenous fistulas. J Neurosurg Pediatr 7:637-642, 2011

16. Sheth SA, Potts MB, Sneed PK, Young WL, Cooke DL, Gupta N, et al: Angiographic features help predict outcome after stereotactic radiosurgery for the treatment of pediatric arteriovenous malformations. Childs Nerv Syst 30:241-247, 2014

17. Viñuela F, Fox AJ, Kan S, Drake CG: Balloon occlusion of a spontaneous fistula of the posterior inferior cerebellar artery. Case report. J Neurosurg 58:287-290, 1983

18. Walcott BP, Smith ER, Scott RM, Orbach DB: Dural arteriovenous fistulae in pediatric patients: associated conditions and treatment outcomes. J Neurointerv Surg 5:6-9, 2013

19. Walcott BP, Smith ER, Scott RM, Orbach DB: Pial arteriovenous fistulae in pediatric patients: associated syndromes and treatment outcome. J Neurointerv Surg 5:10-14, 2013

20. Weon YC, Yoshida Y, Sachet M, Mahadevan J, Alvarez H, Rodesch G, et al: Supratentorial cerebral arteriovenous fistulas (AVFs) in children: review of 41 cases with 63 non choroidal single-hole AVFs. Acta Neurochir (Wien) 147:17-31, 2005

21. Yen CP, Ding D, Cheng CH, Starke RM, Shaffrey M, Sheehan J: Gamma Knife surgery for incidental cerebral arteriovenous malformations. J Neurosurg 121:1015-1021, 2014

\section{Author Contributions}

Conception and design: Albuquerque, Zaidi. Acquisition of data: Zaidi, Kalani. Analysis and interpretation of data: Albuquerque, Zaidi, Kalani. Drafting the article: Albuquerque, Zaidi, Kalani. Critically revising the article: all authors. Reviewed submitted version of manuscript: all authors. Statistical analysis: Zaidi. Administrative/technical/material support: Albuquerque. Study supervision: Albuquerque.

\section{Correspondence}

Felipe C. Albuquerque, c/o Neuroscience Publications, Barrow Neurological Institute, St. Joseph's Hospital and Medical Center, 350 W. Thomas Rd., Phoenix, AZ 85013. email: neuropub@ dignityhealth.org. 